\title{
Servicio en beneficio de la comunidad: una aproximación cuantitativa a la justicia juvenil restaurativa en Chile
}

\author{
Community Service: A quantitative approach to \\ restorative juvenile justice in Chile
}

Daniela Aída Díaz Bórquez ${ }^{l}$

\section{Resumen}

Este estudio observó 67 casos de jóvenes egresados del Programa Servicio en Beneficio de la Comunidad (SBC) para identificar prácticas efectivas en materia de intervención. Mediante una regresión logística fue posible predecir el éxito del SBC en función de las características de la intervención desarrollada por los profesionales.

Los jóvenes que prestan servicios de alta base comunitaria (actividades recreativas/voluntariado) presentarían cinco veces más chances de lograr los objetivos de intervención, respecto de quienes prestan servicios de escaso vínculo comunitario, tales como labores administrativas o restauración de infraestructura pública. Los resultados generan evidencia empírica a favor del potencial restaurativo del SBC cuando permite restituir el vínculo entre el joven y la comunidad, simbólicamente destruido en la comisión del delito.

Palabras clave: servicios comunitarios, integración social, vinculación comunitaria.

\section{Abstract}

This Study observed cases of young graduates to Community Service Program (CBS), in order to identify effective practices in

1 Universidad Alberto Hurtado, Santiago, Chile. E-mail: ddiaz@uahurtado.cl

Este documento presenta una síntesis de la investigación realizada para obtener el grado de Magíster en Sociología. 
the context of this sanction. By a logistic regression method, it was possible to predict the odds to meet the goals of the CBS Program, according to the intervention features developed by professionals.

Young people serving in close connection with the community (recreational / volunteers) are five times more likely to achieve the goals of the intervention, than those serving low-community ties activities (office work / restoration of public infrastructure). The results provide empirical evidence for the CBS restorative potential, when its execution or implementation obtains the young-community bonds restoration, destroyed by the commission of the crime.

Key words: Community service, social integration, community engagement.

El Servicio en Beneficio de la Comunidad (SBC) constituye una propuesta innovadora en materia penal juvenil, puesto que se aproxima a los principios de la justicia restaurativa desde la demanda explícita de vinculación con la comunidad. La integración social sería concebida en esta sanción como un proceso bidireccional que exige la responsabilización del infractor de ley por el daño causado, y al mismo tiempo, el involucramiento de la comunidad en la restitución del vínculo social simbólicamente destruido en la comisión del delito.

En Chile existen 17 proyectos de SBC. El año 2008 egresaron 5.606 jóvenes de las distintas sanciones (medio libre y privativas de libertad) y un 33,4\% de ellos lo hizo desde programas de SBC (Sename, 2009). Al respecto, el Balance Anual de la Ley de Responsabilidad Penal Adolescente (Sename, 2009) expuso las siguientes cifras para el año 2008:

- $\quad 71 \%$ de los casos ingresados al Sistema de ejecución de sanciones son implementados en el medio libre.

- Los SBC vieron excedida su capacidad mensual de atención (882 jóvenes) siendo superados por la demanda y atendiendo un promedio mensual de 1.042 casos.

- 2.306 jóvenes ingresaron a programas de SBC, lo que representa un $26 \%$ de la población total que ingresa a sanciones. 
- Los casos egresados de SBC representaron un $42 \%$ del total egresos de sanciones implementadas en el medio libre.

- En cuanto a causales de egreso, un 37\% de los jóvenes que egresó del SBC lo hizo por cumplimiento de la sanción. Este porcentaje de "éxito" resulta superior al de las sanciones privativas de libertad y al de otras sanciones del medio libre, como la Libertad Asistida Especial (11,9\%).

Considerando estos antecedentes resulta pertinente preguntarse: ¿Cómo se relacionan las características de la intervención desarrollada por el programa del SBC con el cumplimiento de la sanción por parte de los jóvenes? Este estudio pretende identificar factores relacionados al cumplimiento del SBC, analizando las características de la intervención que influyen en el éxito de la sanción. La hipótesis a la base postula que intervenciones intensivas, sensibles a las características de los jóvenes y de alta base comunitaria, otorgarán mayores probabilidades de éxito a la intervención, impactando positivamente el cumplimiento del SBC por parte de los adolescentes.

Antecedentes Teóricos: justicia restaurativa y Servicios en Beneficio de la Comunidad

La justicia restaurativa puede definirse como un paradigma de justicia que enfatiza que la reparación del daño causado o revelado por la conducta delictiva se logra de mejor manera a través de procesos cooperativos que incluyen a todos los involucrados (Confraternidad Carcelaria, 2005). El paradigma surge en Nueva Zelanda en el intento por incorporar la lógica de resolución de conflictos utilizada por pueblos originarios, que asumen que el costo de excluir de la comunidad a quien infringía la norma resulta perjudicial para la supervivencia del grupo, por lo que se le obliga al infractor a reparar el daño causado, rehabilitándolo en ese acto (Kemelmajer, 2005). 
Aunque en Chile los SBC son una sanción más del Sistema de Justicia Juvenil y no una alternativa extrajudicial de resolución de conflictos, es posible identificar algunas nociones restaurativas en su funcionamiento. Reinserción social, responsabilización por las consecuencias de los propios actos y restauración como indemnización simbólica del daño causado a la comunidad (Umbreit, 1998; Walgrave, 1999), son principios restaurativos que se observan con fuerza en la lógica socioeducativa de la justicia juvenil en Chile, y particularmente en el SBC.

Los estudios cuantitativos de efectividad de la justicia restaurativa generan evidencia diferenciada. La experiencia del Reino Unido, por ejemplo, ha avalado la implementación de políticas restaurativas siempre que se tenga en cuenta la particularidad de su funcionamiento, observando mayor efectividad (disminución de reincidencia) en población juvenil que en adultos y al focalizarse en delitos de baja gravedad, "restorative justice works differently on different kind of people" (Sherman \& Strang, 2007, p. 8).

Bergseth y Bouffard (2007) analizaron los efectos de la justicia restaurativa estadounidense comparando jóvenes infractores egresados del sistema judicial restaurativo (SJR) con adolescentes egresados del sistema judicial tradicional (SJT). Al observar la prevalencia de contactos policiales posteriores al paso por ambos sistemas, existen diferencias significativas que se mantienen constantes en el tiempo: un 30\% de los jóvenes egresados del SJT vuelve a tener contacto con el sistema policial y lo hace en menor tiempo que la población egresada del SJR (con un 12\% de prevalencia). La gravedad de los delitos que origina el contacto policial es menor en jóvenes egresados del SJR que en el grupo control, aunque esta relación solo resulta significativa durante el primer año de seguimiento. Además, los jóvenes egresados del SJR evidenciarían un ritmo de prevalencia de la conducta delictual más lento que el grupo control y con menor frecuencia de reincidencia.

La evidencia sobre la efectividad de los SBC es escasa y la atención tiende a dirigirse a las necesidades de los sujetos privados de libertad por sobre aquellos que cumplen condenas en el medio libre (Redondo, 2000). 
No obstante, la literatura internacional observa que ni la prisión ni los trabajos comunitarios resultan en sí mismos sanciones rehabilitadoras o inhibidoras de reincidencia, puesto que, a decir de Blay (2006), la efectividad estaría dada por los contenidos educativos de las sanciones. Con todo, la evidencia indica que los SBC tienen mayor potencial rehabilitador que penas cortas privativas de libertad.

Un estudio de Aebi, Killias y Ribeaud (2000) midió el potencial del SBC para evitar reincidencia, asignando aleatoriamente a condenados a penas privativas de libertad de corta duración a cumplir condena en SBC. Según fuentes policiales, el segundo grupo era menos propenso a reincidir que el grupo control que cumplió penas privativas de libertad. El SBC tendría un efecto positivo, ya que ayudaría a los penados a responsabilizarse por su condena, siendo percibida como consecuencia del propio comportamiento y no como culpa de otros actores del sistema judicial. Coherente con ello, estudios de efectividad de SBC en población penal adulta del Reino Unido focalizados en medir cambios conductuales, observan que los trabajos comunitarios favorecen significativamente la disminución de actitudes y percepciones pro-delictivas de los sujetos penados (Rex \& Gelsthorpe, 2002). Los efectos positivos del SBC se atribuirían a la percepción de utilidad que tiene el trabajo desarrollado para beneficiarios y sancionados (Blay, 2006; McIvor, 1991; Rex \& Gelsthorpe, 2002).

Ahora bien, en cuanto a justicia juvenil los SBC han demostrado consecuencias positivas en el futuro de los jóvenes condenados, quienes tendrían mayores probabilidades de permanencia escolar e inserción laboral que aquellos sentenciados a penas tradicionales, recibiendo además la influencia positiva del proceso extrajudicial en su autoestima, al sentirse “útiles” para la sociedad (Schneider, 1986).

En el intento reciente de observar parámetros estándar de efectividad, un estudio de meta-análisis desarrollado en Reino Unido estableció siete criterios de efectividad para programas de Intervención en Justicia Juvenil, tres de ellos aplicables específicamente a la realidad chilena de los SBC (Stephenson, Giller \& Brown, 2007): 
- Sensibilidad de la intervención profesional al estilo y estrategia de aprendizaje de cada joven, conocida como "responsividad" desde el enfoque de riesgo, necesidad y capacidad de respuesta desarrollado por Andrews y Bonta $(2006)^{2}$.

- Base comunitaria de los programas, para que el aprendizaje asociado a la sanción tenga lugar en contextos significativos y cercanos a la experiencia de vida del joven.

- Intensidad y duración de la intervención, que debe ser periódica y suficiente para lograr sus objetivos.

Es necesario señalar que los criterios restantes correspondientes a clasificación de riesgo de reincidencia, focalización de la intervención en necesidades criminógenas, modalidad, e integralidad de la intervención, no fueron incluidos en este estudio ya que no existían registros de información que permitiesen levantar indicadores cuantitativos para observar el comportamiento de estos factores.

Si bien los criterios de efectividad son genéricos y están respaldados por altos estándares de validación metodológica, la efectividad de un programa siempre está determinada por su contexto. Esta investigación observó indicadores de medición de sensibilidad, base comunitaria e intensidad de la intervención realizada por los SBC en el contexto nacional, para determinar su efecto sobre el cumplimiento de la sanción por parte de los jóvenes.

\section{Metodología}

Se estudiaron casos egresados del Programa de SBC implementado por la Corporación Opción, Organismo Colaborador del Servicio Nacional de Menores y ejecutor del Programa de SBC en la Zona Suroriente de Santiago. Se consideraron egresos del período Octubre 2007 - Julio 2009,

2 Esta teoría centra su atención en factores protectores y de riesgo a los que se ven expuestos los individuos durante su desarrollo y que, como variables personales y contextuales, influyen en el desarrollo de conductas delictuales. Existirían factores de riesgo estático, no modificables (como la historia de vida, edad, etc.) y factores de riesgo dinámicos o modificables que debiesen orientar la intervención (actitudes y pensamiento pro-delictivo, presencia de grupos de pares de influencia negativa, etc.) (Andrews \& Bonta, 2006). 
seleccionando una muestra no probabilística que inicialmente fue de 89 casos. Para efectos del análisis explicativo se excluyeron aquellos casos sin información suficiente en las variables de interés y la muestra se redujo a 67 casos: entre hombres y mujeres, 36 de los cuales egresan del programa por cumplimiento del SBC y 31 por incumplimiento.

Se excluyeron casos críticos de incumplimiento de la sanción por su limitada exposición a la intervención del programa, considerando casos con un mínimo de tres meses de permanencia en el SBC, ya que para distinguir efectos de la intervención es necesario que los jóvenes se hayan encontrado expuestos a ella por un período razonable de tiempo. Particularmente, los casos críticos responden a jóvenes que presentan baja adhesión al programa e inasistencia a reuniones de ingreso, por lo que no existen registros exhaustivos de sus antecedentes.

Además, se excluyeron aquellos casos egresados por motivos distintos al cumplimiento o incumplimiento de la sanción, asumiendo las consecuentes implicancias para la representatividad del estudio. En este sentido, se reconoce cierto sesgo de selección, ya que el alcance de las estimaciones estadísticas se ve limitado por la imposibilidad de capturar la totalidad de la variabilidad de factores asociados al incumplimiento del SBC, sobre todo por la exclusión de casos extremos de incumplimiento cuya inclusión resultaría insostenible.

En términos de datos y procedimientos, se utilizaron registros institucionales del período Julio 2008 - Julio 2009, disponibles en carpetas de sistematización individual del proceso de cada joven durante el desarrollo del SBC. Probablemente el tipo de información disponible y el acceso a ella sea uno de los aspectos más complejos para el desarrollo de investigación en esta materia, dado el carácter cualitativo de los sistemas de registro que utilizan los programas ${ }^{3}$.

Se creó un instrumento para estandarizar la información cualitativa de los registros y generar una base de datos a partir de la sistematización de los registros individuales que cada profesional lleva de los casos atendidos.

3 Tema profundizado en Díaz, D. El desafío de la efectividad en la justicia penal juvenil, Revista de Trabajo Social (82): 67-76 Agosto 2012. 
Se consideraron antecedentes formales estandarizados (protocolos de acuerdo entre el joven y el profesional a cargo del caso, existencia de plan de intervención individual, registro de entrevistas mensuales con el joven y su familia) y documentos jurídicos asociados a la causa penal (informes de ingreso al SBC emanados desde Tribunales e informes de avance de la intervención enviados por el programa a Tribunales). Adicionalmente, se utilizaron datos del Sistema Judicial para acceder retrospectivamente a las trayectorias delictuales de los jóvenes, observando antecedentes penales de la Defensoría Penal Pública (DPP) entre Junio 2007 y Junio 2009. La recolección de información fue apoyada por los profesionales a cargo de la intervención para los casos estudiados. Cabe señalar que se protegió la identidad de los jóvenes, cuidando el carácter confidencial de la información y dando a conocer exclusivamente estimaciones generales de los datos, a fin de resguardar los parámetros éticos de la investigación social.

La Tabla 1 describe las variables observadas, incluyendo variables sociodemográficas (sexo y edad) establecidas como control para neutralizar sus efectos en la estimación de las chances de cumplimiento del SBC, ya que no se consideraron como criterio de selección de la muestra. Respecto al sexo, el 8,6\% de los ingresos anuales al Sistema de Sanciones son mujeres (Sename, 2009) y contarían con trayectorias delictuales de mayor consideración, siendo más propensas a incumplir la sanción de SBC. En cuanto a la edad, la relación edad - conductas delictuales es significativa (Akers \& Lee, 1999; Andrews $\&$ Bonta, 2006) y se busca neutralizar sus efectos en el cumplimiento del SBC. 
Tabla 1

Variables incorporadas al modelo de regresión logístico para explicar el cumplimiento del SBC.

\begin{tabular}{|c|c|c|}
\hline Variable & Descripción & Valores \\
\hline \multirow{2}{*}{$\begin{array}{l}\text { Variable } \\
\text { dependiente: } \\
\text { cumplimiento } \\
\text { del SBC. }\end{array}$} & \multirow{2}{*}{$\begin{array}{l}\text { Logro de los objetivos plantea- } \\
\text { dos en la intervención. Supone la } \\
\text { conclusión efectiva de la condena } \\
\text { producto del cumplimiento de las } \\
\text { horas de servicios comunitarios } \\
\text { decretadas judicialmente. El egre- } \\
\text { so del SBC "por cumplimiento" } \\
\text { no necesariamente implica el cese } \\
\text { de la conducta delictual, sino que } \\
\text { remite exclusivamente al cumpli- } \\
\text { miento de la sanción establecida. }\end{array}$} & 0 = Incumplimiento SBC. \\
\hline & & 1 = Cumplimiento SBC. \\
\hline \multirow{2}{*}{$\begin{array}{l}\text { Variable } \\
\text { independiente: } \\
\text { intensidad de } \\
\text { intervención. }\end{array}$} & \multirow{2}{*}{$\begin{array}{l}\text { Frecuencia de las intervenciones } \\
\text { directas realizadas con el joven } \\
\text { durante su primer mes de per- } \\
\text { manencia en el programa (en- } \\
\text { trevistas individuales, familia- } \\
\text { res, visitas domiciliarias). }\end{array}$} & $\begin{array}{l}0 \text { = Mínimo señalado por Sena- } \\
\text { me para el funcionamiento del } \\
\text { SBC ( } 2 \text { intervenciones). }\end{array}$ \\
\hline & & $\begin{array}{l}\text { 1= Intensidad superior al mínimo } \\
\text { señalado por Sename ( } 3 \text { o más in- } \\
\text { tervenciones). }\end{array}$ \\
\hline \multirow{2}{*}{$\begin{array}{l}\text { Variable } \\
\text { independiente: } \\
\text { sensibilidad de } \\
\text { la intervención } \\
\text { a particularida- } \\
\text { des del joven. }\end{array}$} & \multirow{2}{*}{$\begin{array}{l}\text { Plan de trabajo establecido } \\
\text { conjuntamente entre joven y } \\
\text { delegado para el desarrollo de } \\
\text { la sanción. La elaboración con- } \\
\text { junta del documento considera } \\
\text { intereses y actividades conven- } \\
\text { cionales realizadas por el joven } \\
\text { para determinar el servicio a } \\
\text { prestar. }\end{array}$} & $\begin{array}{l}0=\text { Plan de trabajo elaborado } \\
\text { exclusivamente por el delegado. }\end{array}$ \\
\hline & & $\begin{array}{l}1=\text { Plan de trabajo elaborado } \\
\text { conjuntamente por joven y de- } \\
\text { legado. }\end{array}$ \\
\hline \multirow[t]{2}{*}{$\begin{array}{l}\text { Base comu- } \\
\text { nitaria de la } \\
\text { intervención. }\end{array}$} & \multirow{2}{*}{$\begin{array}{l}\text { Variable independiente que res- } \\
\text { ponde al tipo de servicio presta- } \\
\text { do a la comunidad en el marco } \\
\text { de la sanción. Se asume que } \\
\text { prácticas de mayor base comu- } \\
\text { nitaria promueven el vínculo } \\
\text { del joven con la comunidad du- } \\
\text { rante la ejecución de la sanción. }\end{array}$} & $\begin{array}{l}0=\text { SBC de escasa base comuni- } \\
\text { taria (mantención infraestruc- } \\
\text { tura o trabajo administrativo). }\end{array}$ \\
\hline & & $\begin{array}{l}1=\text { SBC de alta base comunita- } \\
\text { ria (servicios de ayuda social o } \\
\text { voluntariado). }\end{array}$ \\
\hline
\end{tabular}

Fuente: Elaboración propia. 
Respecto a las técnicas de análisis de datos, se realizó un análisis descriptivo de la muestra estudiada y de las características de la intervención desarrollada por el programa de SBC, para luego establecer un análisis inferencial-correlacional entre las características de la intervención (sensibilidad, intensidad, base comunitaria) y la variable dependiente cumplimiento del SBC. Las variables de intervención que resultaron estadísticamente significativas para el cumplimiento del SBC fueron incluidas como variables dicotómicas para indicar presencia o ausencia de un atributo en el modelo explicativo. Finalmente, se realizó una regresión logística multivariable para estimar el cumplimiento de la sanción en función de las características de la intervención desarrollada por el programa SBC.

\section{Resultados}

\section{Caracteristicas de los jóvenes en $S B C$}

Un $64 \%$ de los jóvenes tiene entre 16 y 17 años y un $25 \%$ es mayor de edad (18 a 20 ańos), que habiendo cometido delitos previo a los 18 ańos, fue condenado a SBC en el marco de la Ley de Responsabilidad Penal Adolescente.

Considerando el tipo de sistema de salud como variable proxy del nivel socioeconómico, se observa que no existen jóvenes afiliados al sistema privado. La mayoría de ellos (63\%) se concentra en el grupo indigente o "tramo A" de Fonasa y un 19\% lo hace en el "tramo B" con ingresos inferiores o iguales a $\$ 165.000$ (Fonasa, 2010). Así, es posible afirmar que alrededor del $80 \%$ de los jóvenes proviene de familias de escasos recursos o en situación de extrema pobreza, por lo que la población objetivo del SBC es bastante uniforme en términos socioeconómicos y relativamente homogénea en cuanto a factores de riesgo estructurales asociados a pobreza (Trudel \& Puentes-Neuman, 2000).

Preliminarmente, es posible señalar que el nivel socioeconómico actuaría como criterio previo de selección de los jóvenes sancionados por el Sistema Penal Juvenil, de manera coherente con lo planteado por Ramos y Guzmán (2000) antes de la entrada en vigencia de la Ley de Responsa- 
bilidad Penal Adolescente, donde muchos de los jóvenes de nivel socioeconómico medio y alto que cometían delitos o faltas de mediana gravedad como el hurto en los supermercados, eran rescatados en las comisarías por sus padres sin judicializarse la infracción, situación que no ocurría en caso de jóvenes de escasos recursos.

Si bien es cierto que las condiciones de pobreza pueden ser un factor de riesgo relacionado a la conducta delictual (Trudel \& PuentesNeuman, 2000), cabe señalar que a cuatro años de la implementación de la Ley de Responsabilidad Penal Adolescente (2005) no existe evidencia que asegure la judicialización de delitos de baja gravedad cometidos por jóvenes de niveles socioeconómicos medios y altos, obstaculizando la observación de los efectos del nivel socioeconómico en el cumplimiento de la sanción, al menos en el caso particular del SBC. Esto se vuelve relevante cuando esta sanción se orienta a delitos de baja gravedad que resultan transversales al nivel socioeconómico, particularmente en el caso de población adolescente como lo indica la criminología del desarrollo.

Respecto del tipo de delito, "hurto simple" y "robo por sorpresa" constituyen el 50\% de las causales de ingreso a la sanción de SBC, los delitos "porte de armas" y "receptación" de especies representan un 20\% de las causales de ingreso estudiadas, y el porcentaje restante refiere a "otros delitos" asociados a distintos tipos de lesiones. El análisis observó que no existe una relación estadísticamente significativa entre tipo de delito y cumplimiento/incumplimiento de la sanción de SBC, lo que se explica por causales de ingreso relativamente homogéneas asociadas a delitos de baja gravedad. Esto resulta consistente con el sentido de la sanción y los criterios de efectividad observados en estudios internacionales, que hacen del SBC una sanción idónea para infractores penalizados por delitos de baja gravedad.

En cuanto a trayectoria delictual se observa que un $74 \%$ de los jóvenes registra causas anteriores al ingreso al SBC por infracción a la Ley Penal en la Defensoría Penal Pública (DPP), mientras que solo el 26\% de ellos figura sin antecedentes delictuales posteriores a la implementación de la Ley No 20.084. Llama la atención que un 42\% de los casos registre 
incluso tres o más causas en la DPP, que no necesariamente concluyen en sanción, pero podrían indicar presencia de conductas de riesgo.

Un 48\% de los jóvenes no registra condenas anteriores al ingreso al SBC; mientras que un $11,2 \%$ de ellos registra dos condenas a alguna sanción del sistema. Un 6,7\% de los casos presenta condena por tres o más delitos previos al ingreso al SBC, porcentaje que podría ser considerado como población con algún grado de compromiso delictual, de manera contradictoria con el sentido de la sanción que busca focalizar la intervención en población primeriza e infracciones de baja gravedad.

En cuanto a factores protectores, específicamente escolaridad, solo un $8 \%$ de los jóvenes de la muestra estudiada presenta situación escolar al día, correlativa a su edad. En un $62 \%$ de los casos existe presencia de actividades pro-sociales (escolares o laborales), lo que actuaría como factor protector considerando que los jóvenes que se encuentran realizando alguna de estas actividades presentaron mayores porcentajes de cumplimiento del SBC (64\%), respecto de aquellos sin actividad (39\%).

\section{Características de la intervención desarrollada por el programa $S B C$}

Un $72 \%$ de los jóvenes que realiza el SBC presta servicios en su comuna de residencia, mientras que un $20 \%$ incumple y deserta del programa antes de alcanzar a definirse el servicio a prestar para ejecutar la sanción.

Respecto al tipo de servicio prestado a la comunidad, la mayoría de los jóvenes (70\%) presta servicios como mantención de infraestructura y apoyo a labores administrativas realizadas por las instituciones contrapartes, otorgando prestaciones que no necesariamente implican establecer un vínculo con la comunidad. Por el contrario, el 30\% restante realiza actividades de voluntariado social o participación en gestión de actividades recreativas organizadas por instituciones contraparte, prestando servicios de alta base comunitaria que fomentan el vínculo cara a cara del joven con su entorno. Este tipo de servicios es de vital importancia para el cumplimiento de la sanción, ya que como manifiestan los profesionales del programa, en muchos casos la relación establecida a través de estas actividades 
se sostiene en el tiempo una vez cumplida la sanción, transformándose en un factor protector.

A fin de sistematizar el proceso de intervención, la Corporación Opción ha establecido distintos hitos a cumplir durante la implementación de la intervención, lo que permitió observar la sensibilidad de esta a las particularidades de los jóvenes, probablemente uno de los criterios más difíciles de medir. La intervención se inicia con una entrevista de ingreso en que el profesional a cargo del caso expone al joven su situación judicial, explicando características y responsabilidades de la sanción, situación que es formalizada en la firma de un protocolo de acuerdo. Posteriormente, y una vez que se han explorado los intereses y características del joven, se establece en conjunto un plan de trabajo determinando la forma en que se dará cumplimiento a la sanción. Un $71 \%$ de los jóvenes asiste a la entrevista de ingreso y logra firmar el protocolo de acuerdo. De aquellos que lo hacen, un $73 \%$ egresa de la sanción por cumplimiento. Además, el $75 \%$ de los jóvenes cuentan con un plan de trabajo acordado de manera conjunta con el profesional a cargo del caso, para definir las condiciones de cumplimiento del SBC en base a sus propios intereses y habilidades.

Las variables que midieron la intensidad de la intervención desarrollada por el programa de SBC no presentaron una variabilidad significativa con las consecuentes implicancias para la evaluación de las intervenciones. No es posible medir efectividad en función del número de horas de SBC dictaminado por el Juez, porque el marco legal establece un rango de entre 30 a 120 horas de servicio, pero la mayoría de los casos estudiados (79\%) es sancionado a prestar entre 20 y 30 horas de SBC (Sename, 2009), lo que dificulta la medición de los efectos de la intensidad de la intervención en el cumplimiento de la sanción. Algo similar ocurre con la frecuencia de prestación de servicios determinada conjuntamente por el joven y su delegado, puesto que generalmente los jóvenes prestan servicios 2 o 3 veces por semana en la institución contraparte. Así, como indicador de intensidad de la intervención se utilizó el número de intervenciones directas establecidas por Sename en las Orientaciones Técnicas, para desarrollar estrategias de acompañamiento y supervisión del cumplimiento del acuerdo establecido para prestar el servicio (Sename, 2007). 
Modelo Explicativo: Características de la intervención que explicarian el cumplimiento del SBC

A partir de un modelo de regresión logística, se cuantificaron los efectos de las características de la intervención (intensidad, base comunitaria, sensibilidad a las características del joven) sobre el cumplimiento de la sanción de SBC por parte de los adolescentes. Las variables se incorporan en dos bloques, donde el primero integra las variables control (edad y sexo) y el segundo reúne los indicadores de intervención (plan de trabajo conjuntamente acordado, carácter vinculante del SBC e intensidad de la intervención). El tercer bloque incluye la variable actividad actual, reconocida en la literatura internacional como un importante factor protector ante la conducta delictual e identificada como un factor estadísticamente significativo en regresiones realizadas con los mismos datos para explicar el cumplimiento del SBC a partir de variables individuales de los jóvenes.

Dado el carácter aplicado de este estudio y el interés por levantar evidencia cuantitativa relevante para la intervención, se excluyeron factores de riesgo de carácter estático que, asociados a la biografía de los jóvenes, no son modificables, porque no resultan sensibles a la intervención. Esto, por cuanto la eficacia de una intervención, se asocia siempre a la selección de factores de riesgo que son susceptibles de cambio (Lipsey \& Derzon, 1998).

La Tabla 2 presenta los resultados del modelo de regresión logística, utilizado para estimar los efectos de las características de la intervención en el cumplimiento de la sanción. 
Tabla 2

Modelo de regresión para la estimación de efectos de la pertinencia de la intervención sobre el cumplimiento del SBC

\begin{tabular}{|c|c|c|c|c|c|}
\hline & Variable & $\begin{array}{c}\text { Categoría / Categoría de } \\
\text { Referencia }\end{array}$ & $\begin{array}{l}\text { Bloque } 1 \\
\text { Exp. }(\beta)\end{array}$ & $\begin{array}{l}\text { Bloque } 2 \\
\text { Exp. }(\beta)\end{array}$ & $\begin{array}{l}\text { Bloque } 3 \\
\text { Exp. ( } \beta)\end{array}$ \\
\hline \multirow{2}{*}{$\begin{array}{l}\text { Variables } \\
\text { Socio-demo- } \\
\text { gráficas } \\
\text { (variables de } \\
\text { control) }\end{array}$} & Sexo & $\begin{array}{l}\text { Hombre. } \\
\text { Categoría de referencia: } \\
\text { Mujer. }\end{array}$ &, 526 & ,920 & 1,180 \\
\hline & Edad & $\begin{array}{l}17 \text { o mayor edad } \\
\text { Categoría de referencia: } \\
14 \text { a } 16 \text { años. }\end{array}$ & 1,497 & 1,516 & 1,774 \\
\hline \multirow{3}{*}{$\begin{array}{l}\text { Variables de } \\
\text { intervención }\end{array}$} & $\begin{array}{l}\text { Sensibilidad } \\
\text { de la Inter- } \\
\text { vención }\end{array}$ & $\begin{array}{l}\text { Plan de trabajo sin partici- } \\
\text { pación del joven. } \\
\text { Categoría de referencia: } \\
\text { Plan de trabajo acordado } \\
\text { entre joven y delegado. }\end{array}$ & & $4,534^{* *}$ & 3,121 \\
\hline & $\begin{array}{l}\text { Base comu- } \\
\text { nitaria SBC }\end{array}$ & $\begin{array}{l}\text { Escasa base comunita- } \\
\text { ria: SBC de mantención } \\
\text { infraestructura y trabajo } \\
\text { administrativo. } \\
\text { Categoría de referencia: } \\
\text { Alta base comunitaria, } \\
\text { SBC de ayuda social o } \\
\text { voluntariado. }\end{array}$ & & $3,621^{* *}$ & $5,015^{* *}$ \\
\hline & $\begin{array}{l}\text { Intensidad } \\
\text { Interven- } \\
\text { ción } \\
\end{array}$ & $\begin{array}{l}\text { Mínimo } 2 \text { intervenciones. } \\
\text { Categoría de referencia: } 3 \\
\text { o más intervenciones. }\end{array}$ & & $2,905^{*}$ & $3,644^{*}$ \\
\hline $\begin{array}{l}\text { Factor pro- } \\
\text { tector } \\
\text { individual }\end{array}$ & $\begin{array}{l}\text { Actividades } \\
\text { convencio- } \\
\text { nales }\end{array}$ & $\begin{array}{l}\text { Ausencia actividades } \\
\text { convencionales. } \\
\text { Categoría de referencia: } \\
\text { Presencia actividades } \\
\text { convencionales (trabajo o } \\
\text { estudio). }\end{array}$ & & & $5,299^{* *}$ \\
\hline \multicolumn{2}{|c|}{ Constante } & & 2,160 & $0,153^{*}$ &, $040^{* *}$ \\
\hline \multicolumn{2}{|c|}{ R2 de Nagelkerke } & & 0,041 & 0,370 & 0,456 \\
\hline \multicolumn{2}{|c|}{ Hosmer y Lemeshow } & & 0,509 & 0,694 & 0,815 \\
\hline \multicolumn{2}{|c|}{ Likelihood chicuadrado } & & 2,093 & 19,664 & 6,251 \\
\hline \multicolumn{2}{|c|}{ grados de libertad } & & 2 & 3 & 1 \\
\hline valor $p$ & & & 0,351 & 0,000 & ,012 \\
\hline
\end{tabular}

${ }^{* *} \mathrm{p}<0,05 ;{ }^{*} \mathrm{p}<0,01$. 
Con una adecuada bondad de ajuste ${ }^{4}$ el modelo logra explicar el cumplimiento del SBC en función de las características de la intervención desarrollada por el programa. En cuanto al comportamiento de las variables de control en los casos estudiados, sexo y edad (bloque 1) no presentan una relación estadísticamente significativa con las chances de cumplir el SBC. En términos generales, los coeficientes exponenciales $\beta$ observados en la Tabla 2 indican que la escolaridad de los jóvenes y la base comunitaria de la intervención, son las variables explicativas de mayor relevancia, ya que generan diferencias importantes en las posibilidades de cumplir con la sanción. Los jóvenes que realizan actividades convencionales como trabajar o estudiar, tienen 5,2 veces más chances de desempeñar exitosamente los SBC y egresar del programa por cumplimiento, versus aquellos jóvenes que no presentan este factor protector durante el desarrollo de la intervención. De manera coherente con la evidencia internacional, los efectos de la actividad convencional sobre el cumplimiento de la sanción son positivos y estadísticamente significativos.

La sensibilidad de la intervención a las características del adolescente observada a través del plan de trabajo, aporta antecedentes interesantes en tanto materializa la consideración de las particularidades del adolescente y su responsabilización en la implementación de la sanción, en la medida en que el servicio a prestar y el modo de hacerlo, se definen de manera conjunta entre adolescente y profesional a cargo del caso. El modelo observa que los jóvenes que participaron en la elaboración del plan de trabajo tienen 3,1 veces más posibilidades de cumplir con la sanción de SBC, respecto de aquellos casos donde la intervención desplegada por el programa es diseñada exclusivamente por el delegado. En este sentido, una intervención sensible a las características del joven (intereses, habilidades, capacidades, etc.) y capaz de involucrarle en la planificación del cumplimiento de la sanción, tendría un impacto positivo en los resultados de la misma. De manera coherente con la literatura, el potencial rehabilitador

4 La verosimilitud del modelo reflejada en el estadístico likelihood indica que las variables incluidas en los bloques 2 y 3 mejoran significativamente la verosimilitud del modelo, con valores $\mathrm{p}<0,05$. Los niveles de especificación y bondad de ajuste observados en la prueba de Hosmer y Lemeshow definen la correcta clasificación de los casos en función de la variable dependiente y las pruebas chi-cuadrado reflejan un modelo de regresión bien especificado al no resultar estadísticamente significativas. 
del SBC radicaría en el empleo que da la sanción a las capacidades del penado (West, 1976), donde un plan de trabajo de común acuerdo permite focalizar la intervención en las habilidades de los jóvenes por sobre sus carencias.

No obstante, la presencia del plan de trabajo de común acuerdo pierde efecto sobre el cumplimiento del SBC al incluir la variable actividad convencional como se observa en el bloque No 3. Preliminarmente, podría afirmarse que la sensibilidad a las características del joven (materializada en el plan de trabajo) se relacionaría a la intensidad de la intervención, puesto que intervenciones de mayor frecuencia facilitarían el establecimiento de un plan de trabajo de común acuerdo 5 .

Ahora bien, pese a que estas variables se encontrarían relacionadas, resulta teóricamente relevante estimar la probabilidad de cumplimiento del SBC a partir de las características de la intervención, puesto que permite identificar áreas estratégicas para el logro de buenos resultados, aplicando criterios de efectividad reconocidos por la evidencia internacional en el estudio de intervenciones en materia de justicia juvenil.

En cuanto a la intensidad de la intervención, los análisis realizados en función del número de entrevistas directas con el joven, permiten señalar que esta tiene efectos positivos en el cumplimiento del SBC. Cuando la intervención desarrollada por el programa tiene una intensidad superior a la frecuencia mínima definida por Sename para contactos directos con el joven, hay 3,6 veces más chances de cumplir con la sanción, respecto de casos con intervenciones menos intensivas. Intervenciones más intensivas aumentan las posibilidades de cumplir con los objetivos de la sanción, dependiendo siempre del contenido de la intervención. Así por ejemplo, intervenciones de escaso contenido educativo ejecutadas de manera intensiva a lo largo del tiempo pueden influir en el sentido reparatorio que tiene la sanción para el adolescente, transformando el servicio en un trámite desagradable que pierde su sentido original.

Tan importante como la frecuencia de la intervención son las características y el contenido de la sanción, donde la efectividad de los SBC

5 Pese a que la correlación resulta estadísticamente significativa, no existe multicolinealidad perfecta entre ninguna de las variables de intervención incluidas en las estimaciones. 
se jugaría en el carácter educativo de las sanciones aplicadas (Blay, 2006). Esto constituye un desafío tanto para la intervención como para los mecanismos de evaluación implementados por Sename, que se han centrado en la gestión, monitoreando los programas de SBC en función de indicadores de frecuencia de las intervenciones (número de contactos con instituciones, número de entrevistas con el joven, etc.) por sobre el contenido de las mismas. Particularmente, si el contenido educativo de la intervención influye en el cumplimiento de la sanción, entonces es necesario complementar control de gestión (¡cuánto se hace?) con control de calidad, levantando criterios de efectividad que permitan evaluar la calidad de la intervención (¿cómo se hace y con qué resultados?).

En este sentido el principal hallazgo del estudio radica en la importancia que asume el tipo de servicio prestado a la comunidad para el cumplimiento de la sanción: las intervenciones de alta base comunitaria -entendidas como aquellas que fomentan servicios que promueven un vínculo restaurativo entre el joven y la comunidad- resultan considerablemente efectivas. En efecto, más allá de la observación de otras variables, el tipo de servicio prestado por el joven a la comunidad es trascendental para el cumplimiento de los objetivos de la intervención: los jóvenes que prestan servicios de alta base comunitaria, tienen cinco veces más chances de egresar por cumplimiento del programa de SBC, respecto de los casos donde el servicio prestado no implica mayor vinculación con la comunidad. Particularmente, la mantención de infraestructura pública y el apoyo a labores administrativas, disminuyen las posibilidades de cumplir con la sanción.

\section{Discusión y conclusiones}

Los resultados de este estudio son coherentes con la evidencia internacional que indica que la base comunitaria tiene influencia positiva sobre la efectividad de las intervenciones. Los Programas de SBC resultan más efectivos cuando la intervención se implementa en contextos comunitarios relevantes en la experiencia de los jóvenes, y en función de desafíos cotidianos a resolver (Stephenson et. al., 2007). La efectividad de la san- 
ción se jugaría en su contenido y su capacidad de restablecer el vínculo del joven con la comunidad.

La restitución del vínculo social resulta particularmente relevante al tratarse de justicia penal juvenil, puesto que en la etapa de la adolescencia la necesidad de ser valorado por otros y de generar un sentido de pertenencia se intensifica (Bazemore \& Umbreit, 1997). Las sanciones de alta base comunitaria, ejecutadas en una relación cara a cara con agentes comunitarios, otorgan al adolescente la posibilidad de re-significar el vínculo social, con una comunidad que ha representado experiencias de marginación y exclusión social, que resultan evidentes al observar variables como la trayectoria escolar y el nivel socioeconómico de los jóvenes.

La efectividad de los servicios de alta vinculación comunitaria se explicaría en la idea del SBC como una sanción o solución que articula la responsabilidad personal del infractor y la responsabilidad de la comunidad (Umbreit, 1998). Intervenciones de carácter vinculante facilitarían la reparación del vínculo social al considerar las necesidades y capacidades del joven que cumple la sanción, y al mismo tiempo los intereses de la comunidad representados por las instituciones que actúan como contraparte en cumplimiento del SBC.

Desde esta perspectiva, intervenciones basadas en actividades recreativas, de voluntariado o de ayuda social, responderían a los principios de la justicia restaurativa, puesto que se centran en la reparación del daño causado, pero intencionando la reconstrucción de las relaciones sociales. Esta práctica restaurativa ha observado evidencia significativa a nivel internacional en su aporte a la construcción de comunidades y al control social informal de la delincuencia (Bazemore \& Umbreit, 1997).

El programa de SBC estudiado muestra que queda pendiente identificar y sumar a la tarea de la reinserción social a agentes comunitarios de los contextos en que viven los jóvenes atendidos que resulta trascendental y constituye, al mismo tiempo, un desafío permanente para mejorar la efectividad de la intervención. Consistentemente con ello, hallazgos internacionales como los de la Oficina de Justicia Juvenil y Prevención de la Delincuencia en EEUU, insisten en que la implementación de modelos restaurativos en el ámbito de la justicia juvenil requiere del desarrollo de 
nuevos roles para víctimas, ciudadanos e infractores, articulando objetivos de sanción, rehabilitación y seguridad pública (Bazemore \& Umbreit, 1997).

Sociedad y comunidad deberían asumir roles activos en la implementación de las sanciones para poder articular la retribución del daño causado con las metas de intervención. Esto exige a los operadores de los programas focalizar esfuerzos en la tarea de gestión y selección de instituciones contraparte. Si los servicios de alta vinculación comunitaria tienen efectos positivos sobre el cumplimiento de la sanción, entonces el tipo de servicio a prestar en una determinada institución debiese integrarse como criterio prioritario de selección de las contrapartes que apoyan la ejecución de la sanción.

La importancia que adquieren los actores comunitarios en la implementación de programas de SBC es coherente con la noción de stakeholders relevada por la literatura internacional, referente a grupos de interés o actores comunitarios que debiesen sumarse de manera activa al objetivo de la reinserción social en el espacio local, porque pueden afectar y verse afectados por el problema de la delincuencia.

En este sentido, el otorgar un rol importante a los agentes comunitarios implica liderar esfuerzos locales para construir una visión común respecto de la reinserción juvenil mediante los programas de Servicio en Beneficio de la Comunidad: identificar actores clave; alinear misiones y metas de las instituciones (operadores y contrapartes); definir estrategias de intervención; acordar una visión clara de qué se entenderá por éxito en la implementación de la sanción; definir cómo se materializará el carácter socioeducativo de la intervención; intercambiar información; aclarar responsabilidades; y definir funciones para aportar a las metas de reinserción social y justicia restaurativa.

Si se considera que hay una dimensión simbólica en el significado que se atribuye al castigo penal, es posible afirmar que los SBC tienen mayor carácter social que otras sanciones como la libertad asistida o las sanciones privativas de libertad, puesto que es la sociedad en su conjunto la que asume un rol socioeducativo (Redondo, 2000). En este contexto, la efectividad de las estrategias de intervención de los programas de SBC, dependería necesariamente de contar con una comunidad activa en el proble- 
ma de la reinserción social para avanzar hacia sanciones de mayor carácter social y con mayor impacto en la conducta futura de los jóvenes.

Los resultados de este estudio resultan interesantes para la discusión de la efectividad de la intervención desarrollada por los SBC, puesto que es posible observar variables estratégicas, que al ser intencionadas por los programas pueden adquirir un impacto importante sobre el logro de los objetivos de la intervención y el cumplimiento de la sanción por parte de los jóvenes.

Si los programas de SBC resultan más efectivos cuando la intervención se implementa en contextos comunitarios relevantes en la experiencia de los jóvenes, y en función de desafíos cotidianos a resolver (Stphenson et al., 2007); entonces debiese existir especial atención al tipo de servicio comunitario que prestan los jóvenes, promoviendo el vínculo con la comunidad y alejándose de labores como tareas administrativas o de mantención de infraestructura que no asumen mayor vínculo con el contexto.

La base comunitaria de las intervenciones y el nivel y la calidad de los stakeholders e instituciones contrapartes que involucra un programa, pudiesen ser criterios relevantes para evaluar la efectividad de los SBC. En este sentido, los operadores de las sanciones enfrentan la necesidad de levantar estrategias de involucramiento de actores locales que afectan y resultan afectados por el problema del delito, e intensificar esta estrategia de intervención contribuiría a la efectividad en la medida en que fomenta un rol preponderante de la ciudadanía en el control de la delincuencia y permite mejorar los niveles comunitarios de soporte al funcionamiento de los programas de SBC (Marshall, 1999).

Evidentemente, esto implica focalizar recursos económicos y humanos para socializar el quehacer de los programas de SBC y sensibilizar a los actores locales. Desde la perspectiva de los stakeholders, las instituciones contrapartes son mucho más que un escenario de ejecución de la sanción o que instituciones dispuestas a prestar voluntariamente un servicio social para la implementación de sanciones penales. El desafío es hacer que se sientan parte de la tarea de reinserción social y responsables de liderar 
esfuerzos locales para la implementación de intervenciones efectivas. Más que informar el cumplimiento de la sanción, las contrapartes debiesen monitorear la intervención, participando en todo el proceso para asegurar el cumplimiento de sus objetivos.

De otro lado, resguardar la base comunitaria de la intervención y particularmente el tipo de servicio prestado por los jóvenes, permite responder al objetivo de integración social establecido por Sename para los SBC (Sename, 2007). El desarrollo de un vínculo significativo con la comunidad durante el cumplimiento de la sanción, favorece el cambio de la percepción que la propia comunidad tiene respecto de los "jóvenes delincuentes". Esto impactaría en la estigmatización de la población penal juvenil, que de acuerdo a la teoría de la rotulación social constituye un factor importante para la persistencia de la conducta delictiva (Redondo, 2000).

En este contexto, estos resultados constituyen una primera aproximación al potencial restaurativo de los SBC, levantando una serie de temas relevantes para el desarrollo de conocimiento en la materia, tales como la percepción que tienen las instituciones contraparte del SBC, el estudio de casos exitosos que han permanecido vinculados a instituciones contrapartes una vez finalizado el SBC, entre muchos otros.

Múltiples son los esfuerzos realizados por los distintos agentes del sistema judicial, y siempre escasos los recursos disponibles para la intervención en materia de justicia penal juvenil. Con todo, resulta perentorio levantar evidencia que permita especializar intervenciones y mejorar su efectividad, trascendiendo la pregunta clásica de estudios de efectividad en Programas de Intervención de Delincuencia: what works?; para medir efectividad centrada en los contextos locales en los que se implementan las intervenciones: what works, for whom and under what settings? (Guerra, 1998).

Cuando la evaluación realizada por Sename está centrada en el control de gestión, restringe la posibilidad de generar información respecto de la calidad de la intervención para mejorar las prácticas o incluso comparar intervenciones desarrolladas por distintas instituciones (¿qué hacen los programas SCB? y ¿cómo lo hacen?). Este estudio contribuye prelimi- 
narmente identificando indicadores que afectan el resultado de la sanción y que podrían considerarse en la evaluación de los programas: la intensidad de la intervención, su sensibilidad a las características de los jóvenes, y principalmente el tipo de servicio que se presta a la comunidad, son criterios que permiten complejizar la evaluación de la intervención.

Es posible observar que existiría un conocimiento sustentado en la experiencia práctica de los profesionales operadores del programa de SBC, un saber hacer que no necesariamente se ve reflejado en la sistematización de datos o en la producción de conocimientos acerca de la implementación del SBC, lo que resulta indispensable para evaluar la efectividad de la intervención. Así, es necesario dar continuidad a la generación de conocimiento en la materia, lo que desafía a los programas a diseñar formas estandarizadas de registro de las intervenciones para disponer de sistemas de información que faciliten la observación de evidencia cuantitativa.

En este escenario, la disponibilidad de información que permita evaluar y perfeccionar el funcionamiento de las sanciones resulta particularmente relevante si se considera que el SBC constituye una apuesta importante para materializar las posibilidades de la justicia restaurativa en Chile.

\section{Referencias}

Aebi, M., Killias, M. \& Ribeaud, D. (2000). Does community service rehabilitate better than short-therm imprisionment? The Howard Journal, 39, 40-57.

Akers, R. \& Lee, G. (1999). Age, social learning and social bonding in adolescent substance use, Deviant behavior: an interdisciplinary journal, 19, 1-25.

Andrews, D. \& Bonta, J. (2006). The psychology of criminal conduct. Newark, New Jersey, NJ: LexisNexis.

Bazemore, G. \& Umbreit, M. (1997). Balanced and restorative justice for juveniles: a framework for juvenile justice in the 21st century. St. Paul, Minnesota, MN: Center for Restorative Justice \& Peacemaking, University of Minnesota.

Bergseth, K. \& Bouffard, J. (2007). The long-term impact of restorative justice programming for juvenile offenders. Journal of criminal justice, 35, 433-451. 
Blay, G. (2006). La pena de trabajo en beneficio de la comunidad (Tesis doctoral). Recuperada de Tesis en red http://www.tesisenred.net/ bitstream/handle/10803/5084/ebg1de1.pdf? sequence $=1$.

Confraternidad Carcelaria Internacional (2005). ¿Qué es la justicia restaurativa? Trabajo resumen sobre justicia restaurativa, Centro para la Justicia y la Reconciliación, Washington DC, EEUU. Recuperado de http:// www.pfi.org/cjr/espanol/enfoque/whatis/view?searchterm=\%20Justicia\%20Restaurativa

Díaz, D. (2012). El desafío de la efectividad en la justicia penal juvenil. Revista de trabajo social, 82, 67-76.

Fondo Nacional de Salud [Fonasa]. (2010). Tramos de ingreso para atención en modalidad institucional. Recuperado de http://www.supersalud. gob.cl/consultas/570/w3-article-6304.html

Freeman, E. \& David, L. (1983). Stockholders and Stakeholders, a new perspective on corporate governance, California business review, 25(3), 88-105.

Fundación Paz Ciudadana. (2010). Construcción de indicadores de reinserción social de adolescentes infractores de la ley penal. Recuperado de http://www.pazciudadana.cl/docs/pub_20101001131322.pdf

Guerra, N. (1998). Serious and violent juvenile offenders: gaps in knowledge and research priorities. En R. Loeber \& Farrington, D (Eds.), Serious and violent juvenile offenders: risk factors and successful interventions (pp. 389-404). Thousand Oaks, CA: Sage Publications.

Kemelmajer, A. (2005). La tercera vía: la llamada justicia restaurativa reparativa reintegrativa o restitutiva. En S. García. Memoria del Congreso Internacional de Culturas y Sistemas Jurídicos Comparados. I. Derecho Penal. México, D.F.: Instituto de Investigaciones Jurídicas de la UNAM.

Ley No 20.084, Ley de Responsabilidad Penal Adolescente. Diario Oficial de la República de Chile, Santiago, Chile, el 7 de Diciembre de 2005.

Lipsey, M. \& Derzon, H. (1998). Predictors of violent or serious delinquency in adolescence and early adulthood. En R. Loeber \& D.P. Farrington, Serious and violent juvenile offenders: risk factors and successful interventions (pp. 86-105). Thousand Oaks, CA: Sage Publications.

Marshall, T. (1999). Restorative Justice an Overview. A report by the Home Office. Research Development and Statistics Directorate, UK. Recuperado de http://www.homeoffice.gov.uk/rds/pdfs/occ-resjus.pdf

McIvor, G. (1991) Sentenced to serve. The operation and impact of community service by offenders. Aldershot, UK: Avebury. 
Ramos, M. \& Guzmán, J. (2000). La Guerra y la Paz Ciudadana. Santiago de Chile: Lom.

Redondo, S. (Octubre, 2000), Psicología penitenciaria aplicada: los programas de rehabilitación en Europa. Ponencia en las I Jornadas de Tratamiento Penitenciario. Peñíscola, España.

Rex, S. \& Gelsthorpe, L. (2002). The role of community service in reducing reoffending: evaluating pathfinder projects in the UK, Howard Journal, 41(4), 311-325.

Schneider, A. (1986). Restitution and recidivism rates of juvenile offenders: results from four experimental studies. Journal Criminology, 24, 533-52.

Servicio Nacional de Menores. [Sename]. (2007). Orientaciones Técnicas. Servicio en Beneficio a la Comunidad, Departamento de Responsabilidad Penal Juvenil. Santiago de Chile: Autor.

Servicio Nacional de Menores. [Sename]. (2009). Informe Estadístico Balance Anual 2008 Ley de Responsabilidad Penal Adolescente, Departamento de Derechos y Responsabilidad Juvenil. Santiago de Chile: Autor.

Sherman, L. \& Strang, H. (2007). Restorative justice: The evidence (en línea). The Smith Institute, Londres. Recuperado de http://www.sas. upenn.edu/jerrylee/RJ_full_report.pdf

Stephenson, M., Giller, H. \& Brown, S. (2007), Effective practice in youth Justice. UK: William Publishing.

Trudel, M., Puentes - Neuman, G. (2000), The contemporary concepts of at risk children: theoretical models and approaches in the early years. Pan Canadian Education Research Agenda Symposium, Adolescence. Recuperado de http://www.cmec.ca/stats/pcera/symposium2000/ trudel.en.pdf

Umbreit, M. (1998). What is restorative justice? Center for Justice \& Peacemaking. Saint Paul: University of Minnesota.

Walgrave, L. (1999) Restorative juvenile justice: repairing the harm of youth crime. Criminal Justice Press, 327-356.

West, J. (1976), Community Service Orders. En J.S.F. King \& W. Young, (Eds.). Control Without Custody (pp. 68-90) Cambridge: Cambridge Institute of Criminology.

Fecha de recepción: 16 de agosto de 2012.

Fecha de aceptación: 23 de noviembre de 2012. 